WORKING PAPER $\cdot$ NO. 2020-65

Job Loss and Behavioral Change: The Unprecedented Effects of the India Lockdown in Delhi

Kenneth Lee, Harshil Sahai, Patrick Baylis, and Michael Greenstone MAY 2020 


\title{
Job Loss and Behavioral Change: The Unprecedented Effects of the India Lockdown in Delhi*
}

\author{
Kenneth Lee†, Harshil Sahai‡, Patrick Baylis§, Michael Greenstone\|
}

May 15, 2020

\begin{abstract}
On March 24, 2020, the Prime Minister of India announced the world's largest COVID-19 lockdown. We summarize the initial impacts of the lockdown for a representative sample of mostly poor and non-migrant workers in Delhi. Using Facebook mobility data, we show that intra-city movement dropped 80 percent following the announcement. Using microeconomic survey data, collected before and during the crisis, we highlight three patterns. First, the lockdown resulted in significant economic costs, with income and days worked falling by 57 and 73 percent, respectively. Second, the lockdown resulted in widespread compliance with public health directives: mask usage rose by 73 percentage points (pp); time spent indoors increased by $51 \mathrm{pp}$; smoking decreased by $13 \mathrm{pp}$; and handwashing rose by $10 \mathrm{pp}$. Third, the economic impacts of the lockdown were somewhat mitigated by government food assistance, which 36 percent of our sample accessed. Over the first seven weeks of the lockdown, we do not observe alarming levels of hunger, scarcity, access to medical care, or security. Yet in our data, concerns remain about mental health, supply chains, and personal savings, against the backdrop of a rising infection rate. Moreover, it remains to be seen whether public health compliance will persist, as the novelty, fear, and media coverage of COVID-19 subside.
\end{abstract}

\footnotetext{
* We are grateful to Rongmon Deka, Shipra Karan, Ian Pitman, Meghna Singh, Rathan Sudheer, and Ramya Teeparthi for excellent research assistance. All errors are our own. This paper was funded with support from the Tata Center for Development at the University of Chicago.

$\dagger$ Energy Policy Institute at the University of Chicago in India (EPIC India). Email: kennethlee@uchicago.edu, corresponding author.

\$ University of Chicago, Department of Economics. Email: harshil@uchicago.edu.

$\S$ University of British Columbia, Vancouver School of Economics. Email: patrick.baylis@ubc.ca.

|| University of Chicago, Department of Economics. Email: mgreenst@uchicago.edu.
} 


\section{Introduction}

In response to the rapid spread of COVID-19, Prime Minister Narendra Modi announced the world's largest coronavirus lockdown on March 24, 2020, forcing nearly 1.3 billion people to halt virtually all social and economic activities. The policy immediately altered patterns of daily life across India, as factories, non-essential shops, religious buildings, parks, and transportation hubs across the nation were abruptly closed. In this paper, we describe the initial effects of the lockdown on a representative sample of mostly poor and non-migrant workers in Delhi. We focus on how the lockdown affected economic and behavioral outcomes, which we documented, both before and after the onset of COVID-19.

We contribute to a rapidly growing literature describing the economic impacts of COVID19 and the lockdowns and social distancing policies that have been introduced around the world to combat its spread. This literature, along with the daily reports in the news media, have placed a spotlight on the enormous effects of the pandemic on employment, consumption, economic output, and the environment, particularly in developed countries. ${ }^{1}$ However, there is comparatively less information thus far on the microeconomic impacts of the COVID-19 lockdowns in developing countries like India, and even less on compensatory changes in health-related behaviors. ${ }^{2}$ Personal habits and behaviors, like frequent handwashing, social distancing, and mask-wearing in public, have been identified as key actions that individuals could take to reduce the likelihood of infection.

\footnotetext{
${ }^{1}$ For example, in the United States, Baker et al. (2020) show how households radically altered their spending in response to the pandemic; Bartik et al. (2020) describe the impact of COVID-19 on employment and business closures using surveys of small businesses; and Coibion et al. (2020) describe the impacts on employment using surveys of households. In the area of energy and the environment, Burlig, Cicala, and Sudarshan (2020) measure the impact of COVID-19 on reducing electricity consumption and particulate pollution in various countries around the world.

${ }^{2}$ In contemporaneous work in India, Afridi, Dhillon, and Roy (2020) use cross-sectional survey data to reveal large, negative labor impacts for a sample of primarily self-employed and wage laborers in Delhi, a population group that is similar to the one studied in this paper; while Bertrand, Krishnan, and Schofield (2020) use data from a nationally representative survey to show that 84 percent of Indian households experienced a decrease in income and have limited resources to continue coping with the economic situation.
} 
It is widely believed that the impacts of COVID-19 will be the most severe for poor individuals living in the densely populated urban settlements and slums of the developing world. On Day 1 of the India lockdown, for example, some predicted that the resulting economic collapse would be especially devastating for the bottom 50 percent of workers in the informal sector (e.g. Singh et al. 2020). A couple weeks into the lockdown, stories of casual workers in Delhi being forced to survive on food distributed by the government had become common (e.g. Yadav 2020).

There are several reasons why the urban poor would be especially vulnerable to the health and economic impacts of COVID-19, and the policies designed to slow its spread. For instance, the urban poor are likely to face higher rates of transmission risk, not only because they live in overcrowded neighborhoods, but also because the quality of water and sanitation in these neighborhoods tends to be poor (Marx, Stoker, and Suri 2013). In addition, they may face greater mortality risk due to higher rates of pre-existing health conditions (Zheng et al. 2020). Moreover, in Delhi, most people have endured lifelong exposures to extreme levels of air pollution, and higher COVID-19 mortality rates have been associated with higher levels of air pollution (Wu et al. 2020). Finally, the urban poor may be more prone to the economic shocks associated with extreme social distancing mandates, due to their likely employment in wage-paying occupations which tend to require physical work, and low levels of savings which are necessary for smoothing out short-term fluctuations in income.

We report on the initial effects of the India lockdown in Delhi. Using Facebook mobility data, we first show that general intra-city movement dropped to less than 20 percent of normal, following the lockdown announcement. We then shift our focus to our representative sample of 1,392 mostly poor and non-migrant workers in Delhi, consisting of people living in low-income, informal settlements and municipal public bus commuters. Using survey data collected both before 
and during the lockdown, we show that weekly income and days worked fell by 56.5 and 73.3 percent, respectively. While the impacts were equally large across all income quartiles in our sample, they were larger for those employed in daily wage-paying occupations (as opposed to occupations that pay monthly salaries).

At the same time, we observe widespread adoption of recommended public health directives. For instance, mask usage rises by 72.9 percentage points (pp); time spent indoors increases by $50.5 \mathrm{pp}$; smoking decreases by $12.9 \mathrm{pp}$; and regular hand washing rises by $10.0 \mathrm{pp}$. The magnitudes of these effects are large, and in certain cases, rival or exceed the impacts of past interventions (to increase handwashing or encourage mask usage, for example) documented in the development economics literature (see, e.g., Kremer and Zwane 2007; Clasen et al. 2014; Hussam et al. 2019; Baylis et al. 2020). Focusing on mask usage, in particular, we use a combination of survey data and Twitter data to highlight the potential role of extreme fear and unparalleled media coverage in driving these large shifts in behavior.

In our data, we do not observe substantive changes over the course of the lockdown in rates of hunger, product scarcity, ability to access medical care, or security. We also find suggestive evidence that the negative income shocks were mitigated by the Delhi government's widespread food assistance programs, which were accessed by more than one-third of our sample. At the time of this writing, our data suggest that although the India lockdown had a large effect on movement and finances, it did not prevent people from accessing bare essentials. That said, there are warning signs in the data. Relatively high rates of depression, challenges in food supply chains, and dwindling levels of savings pose serious concerns about the cumulative effects of subsequent lockdowns. In addition, it remains to be seen whether the large behavioral changes we observe will 
persist as the novelty, fear, and media coverage of COVID-19 subside, or if access to basic necessities is substantially compromised.

The paper proceeds as follows. In the next section, we offer a timeline of the India lockdown and describe its impact on general intra-city movement in Delhi. We then describe our data collection and discuss the representativeness of our sample. Next, we summarize the leading patterns that emerge from our data. The final section concludes and highlights some of the questions that could be answered through further surveys and research.

\section{Context}

\section{Timing of the India lockdown}

The first case of COVID-19 in India was reported on January 30, 2020. By mid-March, there were just over 100 confirmed cases in the country. As the fear of contagion grew, India acted decisively, and at an early stage relative to its case count. On March 19, Prime Minister Modi announced a 14-hour voluntary public curfew (the Janata curfew) that would take place on March 22. Although the Janata curfew was voluntary, it was believed to be widely followed (as reported by HT Correspondents 2020). Then, on March 24, the Prime Minister ordered all of India to be locked down, severely restricting the movement of and halting nearly all social and economic activities for 1.3 billion people. The lockdown, which was initially planned for 21 days, came into effect just hours following the announcement. On April 14, the Prime Minister extended the lockdown for another 19 days, until May 3; and then on May 1, he extended it again for another 14 days, to May 17. On May 3, the Delhi government announced that it would begin to allow certain relaxations permitted by the Ministry of Home Affairs, including for example, allowing non- 
essential workers and private offices to run at limited capacity, and greater movement of private vehicles. In summary, Delhi residents have effectively been living under lockdown since March 22 , at the time of this writing.

\section{The sharp reduction in intra-city movement}

The India lockdown had a profound impact on social distancing. This can be demonstrated by the effect it had on reducing intra-city movement in Delhi, a plausible proxy for social distancing. To show this, we employ data capturing the movement of over two million Facebook users in Delhi who have enabled location services on their mobile applications. The data, which is made available to researchers and non-governmental organizations through an agreement with Facebook Data for Good, allow us to track intra-city movement at a fine spatial granularity and high temporal frequency (Maas et al. 2019).

Specifically, we aggregate Facebook data from the user-level to 166 administrative regions in the Delhi-National Capital Territory. The data capture the number of users that move from one region to another, during four equally spaced windows each day, representing on average 750 region-to-region movement vectors (ranging from 0.1 to 30 kilometers) every eight hours.

In Figure 1, we demonstrate the reduction in movement over the course of the India lockdown, relative to a baseline that is defined as average movement for the same time-of-day and day-of-week, during the 45 days preceding February 24, 2020. We restrict our focus to a subset of 78 regions for which there is a balanced panel. ${ }^{3}$ The sharp reduction in movement first appeared after March 19, following the announcement of the Janata Curfew. By March 22, movement was down to around 30 percent of normal. By March 24, it was down to between 10 and 20 percent of

\footnotetext{
${ }^{3}$ An unbalanced panel produces qualitatively similar results.
} 
normal, where it remained until May 3, when some of the lockdown restrictions first began to be lifted.

It is likely that the Facebook data is representative of a higher income group than the population we study in this paper, which we describe in detail in the following section. These data correspond to people who use smartphones, the Facebook mobile application, and have access to reliable internet. That said, in our results, we show how respondents in our sample reported similar reductions in social interactions, consistent with the pattern shown in Figure 1.

\section{Data}

\section{Sample selection and data collection}

The sampling frame for this study consists of mostly poor and non-migrant workers living in Delhi, and some of the surrounding urban areas. The sampling frame combines two subsamples established in recent years. The first subsample, which we refer to as "low-income neighborhoods" $(n=3,018)$, was created in 2018 and captures individuals residing in poor, informal settlements across Delhi.

To create this sample, we first consulted the list of Jhuggie Jhopri ("J.J.") Squatter Settlements/Clusters provided by the Delhi Urban Shelter Improvement Board. At the time, this was the only publicly available list of slum clusters or squatter settlements in Delhi. Using this list, we randomly selected a number of sampling points (i.e., locations where enumerators could begin administering in-person surveys) located around the center of each J.J. cluster, in proportion to the cluster's population size. We excluded sampling points that were deemed to no longer be slums or squatter settlements (due to urban development), using a combination of satellite images and inperson checks. This left us with roughly 1,200 sampling points, around which our team of 
enumerators enrolled individuals into our study and administered a brief social and economic questionnaire. These surveys were carried out between October and December 2018.

The second subsample in our sampling frame, which we refer to as "public bus commuters" ( $\mathrm{n}=2,110)$, was created in 2019 and captures individuals who use the public bus system in Delhi, and its neighboring, satellite cities, Gurgaon and Noida. To create this sample, we randomly selected 120 bus stops operated by the Delhi Transport Corporation, 18 bus stops from routes operated by the Noida Metro Rail Corporation, and 79 bus stops from routes operated by Gurgaon Metropolitan City Bus Limited. Our team of enumerators then visited each of these bus stops, repeatedly and at randomized points in the day, to administer a brief social and economic questionnaire. These surveys were carried out between October and December 2019.

In total, there are 5,128 individuals in our sampling frame, including 3,018 individuals from low-income neighborhoods, and 2,110 public bus commuters. We pool together survey data from these samples to represent the pre-COVID-19, or baseline, levels of the economic and behavioral variables.

In the days following the announcement of the India lockdown, we launched additional rounds of telephone surveys, carried out between March 27 and April 19 ("Round 1") and April 25 and May 13 ("Round 2"), respectively. In each round, our team of enumerators contacted individuals from the sampling frame in a randomized order and administered a brief social and economic questionnaire, tailored to capture COVID-19-related outcomes.

In our final dataset, we include 1,392 individuals who are considered to be active participants in the labor force, meaning they were employed in at least one survey round over the course of the study. Note that we surveyed 1,744 individuals in total, including both active participants in the labor force and non-workers (e.g., homemakers, students, etc.), and when we 
include the full sample in our analyses, the findings are the same. That said, for the purposes of this paper, we focus on the workers for whom there is both employment and behavior data. ${ }^{4}$

\section{Sample characteristics and representativeness}

Broadly, the population group we study can be characterized as representative of mostly poor, nonmigrant, male workers (of working age) living in Delhi. In Table 1, column 1, we summarize the key characteristics at baseline for these individuals. The majority of individuals are long-term residents of Delhi ( 87.3 percent) and can thus be considered non-migrants. Only 20.3 percent of our sample reported that they had either migrated or were planning to migrate at some point during the lockdown. Compared to developed country settings, they have relatively low levels of educational attainment (less than half reported completing secondary school) and earn low levels of income (the average weekly income is $\$ 39.46$, which is roughly $\$ 2,052$ per annum). Although 32.4 percent held salaried jobs at baseline (i.e., occupations that pay income on a monthly basis, as opposed to on a daily basis), 29.1 percent held jobs commonly associated with the lower rungs of the income ladder, including auto rickshaw drivers, street vendors, skilled laborers, construction workers, and domestic workers. Baseline levels of health also appear to be poor, with 42.0 percent of respondents in low-income neighborhoods reporting cardiorespiratory health symptoms, possibly the result of living in an environment with extreme levels of air pollution.

In columns 2 and 3, we compare the characteristics of individuals from the low-income neighborhoods to those of the public bus commuters. Respondents in low-income neighborhoods are, on average, more likely to be women (18.6 versus 4.9 percent), less likely to have completed

\footnotetext{
${ }^{4}$ We were able to successfully contact 68 percent of the sampling frame, out of which 66 percent consented to be surveyed. The remainder of the sampling frame could not be enrolled for a number of reasons, including unanswered calls, and non-functioning phone numbers. The full sample is then restricted to individuals whose names matched.
} 
secondary school (35.0 percent versus 65.2 percent), and earn less ( $\$ 28.57$ versus $\$ 53.17$ per week). Despite these differences, the general employment profile is similar, as a substantial share of respondents in both subsamples held occupations that involved low-skill labor and/or required a physical presence.

In Figure 2, we summarize the representativeness of our sample. In Panel A, we plot our sampling locations over a map of the population density in Delhi. The low-income neighborhoods we survey, identified by the red circles, cover some of the most densely populated areas of the city. In panel B, we plot and compare the baseline (i.e., pre-COVID-19) Epanechnikov kernel (bandwidth 10) of weekly income (converted to U.S. dollars) to that of the National Sample Survey (NSS) Delhi sample, inflated to 2019 . At baseline, mean weekly income in our sample is $\$ 39.46$, compared to $\$ 78.84$ in the NSS Delhi sample, which falls in between the 25th and 50th percentile of income, suggesting that we are capturing a slightly poorer segment of the bulk of the overall population.

\section{Results}

We estimate the initial effects of the India lockdown on our sample of mostly poor and non-migrant workers in Delhi through simple pre-post comparisons of key variables. This approach leaves open the possibility of confounding factors. However, we believe there is a limit to these concerns, considering how rapidly the India lockdown was introduced, and the sheer magnitude of the changes we observe. In our view, the only reasonable explanation for the large-scale changes described below is the India lockdown, and the general atmosphere of extreme fear and intense media coverage of COVID-19. 
As of May 13, 2020, roughly seven weeks following the announcement of the India lockdown, three leading patterns emerge from our data: there are large reductions in employment and income; widespread compliance with public health instructions; and limited effects on hunger, access to health care, and security, thus far.

\section{An unprecedented decline in employment and earnings}

In Figure 3, we illustrate the impact of the India lockdown on weekly income for the workers in our sample. In Panel A, we plot the distributions of income at baseline, recorded between 2018 and 2019, income during Round 1 of the lockdown, and income during Round 2 of the lockdown. Compared to baseline, mean weekly income falls by 38.9 percent in Round 1 (i.e., weeks one to four), and then falls a further 77.5 percent in Round 2 (i.e., weeks five to seven), as more and more workers are forced into unpaid leave. By Round 2, weekly income has fallen to zero for 89.2 percent of the sample. Relatedly, average days worked over the past week falls from 5.2 to 1.3 days, a decline of 74.6 percent, by Round 2. Broadly, these results are consistent with those found in contemporaneous work by Afridi, Dhillon, and Roy (2020) and Bertrand, Krishnan, and Schofield (2020).

In Panel B, we report the change in weekly income for different groups of people sorted by their reported occupation at baseline. In the first four weeks of the lockdown, nearly all nonsalaried occupations, including skilled laborers, drivers, street vendors, and domestic workers, experienced losses of 30 percent or more, while salaried workers suffered much less. However, by weeks five to seven of the lockdown, workers across all categories were reporting income losses of more than 60 percent. 


\section{Widespread compliance with public health directives}

The India lockdown has been accompanied by a number of public health messaging campaigns to raise awareness of COVID-19 and encourage people to adopt certain public health behaviors, including regular handwashing, mask-wearing in public, social distancing, and abstinence from smoking and spitting, among other recommendations. In Delhi, these messages have been delivered in various ways. For example, voice recordings have been played at the beginning of all private telephone calls; large billboards have been displayed in the streets; and in some areas, auto rickshaws have been repurposed to patrol the streets while playing health messages through loudspeakers.

In Figure 4, we summarize the impact of the India lockdown on several health-related behaviors that we documented both before and after the onset of COVID-19. In some cases, we observe dramatic changes relative to baseline. Recent mask usage, for example, increased by 72.9 percentage points. The share of respondents stating that they "spent most of yesterday indoors" increased by 50.5 percentage points. Regular handwashing increased by 10.0 percentage points, becoming nearly universal. Even the share of respondents reporting that they do not smoke improved by 12.9 percentage points.

Some of the behavioral gains can be attributed to the short-term bans introduced while the lockdown was underway. On April 15, for instance, the Delhi government temporarily banned the sale of tobacco and announced that everyone would be required to wear a mask in public. ${ }^{5}$ In our data, however, rates of non-smoking and mask usage had already shown dramatic changes in the weeks leading up to April 15, and are similar before and after the introduction of these temporary orders.

\footnotetext{
${ }^{5}$ Anecdotally, the ban on tobacco sales was not well-enforced.
} 
These behavioral effects are notable considering that many defensive health behaviors have low rates of takeup in the developing world (Dupas and Miguel 2017). In certain cases, the effect sizes rival or exceed the impacts of past interventions (to increase handwashing or encourage mask usage, for example) documented in the development economics literature (see, e.g., Kremer and Zwane 2007; Clasen et al. 2014; Hussam et al. 2019; Baylis et al. 2020).

What causes such uniform compliance? The potential role of fear and media in mask usage

In highly polluted cities across the world, face masks had increasingly been seen as a viable defensive measure against the harmful effects of PM 2.5 (see, e.g., Langrish et al. 2012; Cherrie et al. 2018). During the week of November 1, 2019, the peak air pollution period in India, the Delhi government ambitiously distributed five million masks to its residents to help people defend themselves against PM 2.5 concentrations that were surpassing $300 \mu \mathrm{g} / \mathrm{m} 3$ (i.e., 30 times higher than the WHO standard).

In Figure 5, Panel A, we plot mask usage in our sample during the most recent peak period of air pollution (between October and December 2019), and during the India lockdown. Although mask usage increased in the weeks following the government distribution of masks, it did not reach 35 percent of the sample. In contrast, it has become nearly universal during the COVID-19 crisis, despite relatively low levels of air pollution.

It is likely that the behavioral response to the current crisis, relative to the air pollution crisis, has been driven by a combination of factors, chief among them the extreme level of concern and fear about the coronavirus, and the unprecedented level of media coverage of the pandemic. For example, 79.7 percent of respondents in our sample reported feeling "extremely concerned" about COVID-19. In comparison, less than 40 percent of respondents felt this way about air 
pollution during the peak pollution period between October and December 2018. High levels of fear have been well-documented in India. For instance, in some areas, extreme levels of concern led people to not only comply with the recommended health behaviors during the lockdown, but to also form volunteer squads to ensure that others complied (as reported in Gettleman and Raj 2020).

In addition, media coverage of COVID-19 has vastly overshadowed coverage of all other crises in recent memory. In Figure 5, Panel B, we present the daily number of tweets related to COVID-19 and air pollution, produced by 50 leading Indian media and newspaper handles, most of which are based in Delhi (e.g., Times of India, Hindustan Times, etc.). During Delhi's most recent peak air pollution period, there is a sharp increase in pollution-related coverage. At the time, air pollution was one of the most discussed topics in Delhi. Yet the cumulative media coverage of COVID-19 has been many times greater. At their peak, on November 3, 2020, air pollution tweets accounted for 8.8 percent of all tweets, according to our measure. In contrast, on March 27, 2020, just a couple days into the lockdown, COVID-19 tweets accounted for 70.6 percent of all tweets. Based on this measure, from March 25 to May 13, COVID-19 accounted for 55.9 percent of all media coverage, although its share of total coverage has declined. Taken together, these patterns raise the question of whether compliance with public health directives can remain high, even as the novelty, fear, and media coverage of COVID-19 subside once the lockdown is lifted.

\section{Levels of hunger, health care access, and insecurity are not alarming, but concerns remain}

When the lockdown was first announced, many observers were concerned about the emergency issues (e.g., hunger) that could arise if economic activity were to shut down completely, particularly in a setting with such high population density and poverty rates. Migrant laborers, for 
example, were heavily and almost immediately impacted. Just days into the lockdown, tens of thousands of migrant laborers and daily wage workers attempted to leave Delhi and return to their villages of origin, either by bus or on foot, in a migration that resulted in numerous deaths (e.g., as reported in Pal and Siddiqui 2020).

In order to mitigate anticipated hunger needs, the Delhi government set up over 500 assistance centers across the city to provide access to food (typically rice and lentils) for those who needed it. These centers appear to have been well-placed, as the average distance between the individuals in our sample and the nearest government assistance center is only 640 meters. In our data, which covers mostly poor and non-migrant workers in Delhi, it appears that negative impacts on consumption may have been mitigated by this effort. Roughly 34 percent of our sample reported accessing these centers.

In Figure 6, we show the share of respondents in our sample, grouped by income bracket, that reported receiving government assistance (the vast majority of which was in the form of food). The share of recipients is larger for lower income groups (e.g., 46.1 percent of individuals in the lowest quartile of income reported receiving assistance), suggesting that the Delhi government relief efforts were effective in reaching those in greatest need of assistance.

We also do not observe high, or even moderately high, levels of hunger, scarcity, inability to access medical care, or insecurity, as reported in Figure 7. During the first seven weeks of the lockdown, few respondents experienced hunger (3.2 percent reported skipping a meal recently); there were only moderate levels of product scarcity (19.4 percent indicated they were unable to purchase an item); and there were only a handful of reports of crime (4.0 percent stated that they had experienced a recent crime incident). Among individuals who attempted to access medical 
care (or knew of someone personally who attempted to access care), only 4.5 percent reported any challenges.

Nevertheless, whether these patterns will continue even in the immediate future is uncertain. Nearly half of the respondents in our sample reported some difficulties in food supply chains, ranging from mobility restrictions to inadequate quantities, business closures, and high prices. Only 25.2 percent of respondents stated that they had savings remaining, and out of these individuals, 44.1 percent estimated that they would run out of savings soon, a finding that is consistent with the work of Bertrand, Krishnan, and Schofield (2020). We also note concerning levels of mental and emotional well-being during the lockdown. 42.9 percent of respondents reported feeling either depressed, bothered, that they lacked focus, or that everything was an effort, for the majority of the week leading up to the survey.

\section{Discussion}

Taken together, our data suggest that for our representative sample of mostly poor and non-migrant workers in Delhi, the India lockdown imposed harsh financial consequences. At the same time, it induced substantial compliance with public health directives important in limiting the spread of COVID-19, and these changes were potentially driven by high levels of fear and media exposure. Thus far, a worst case scenario (involving alarming rates of hunger and lack of access to health care, for example) has largely been avoided, possibly due to the success of the Delhi government's assistance programs. But with diminishing levels of savings, there remain serious concerns about the near-term future, particularly as infection rates rise. In addition, it remains to be seen whether the positive behavioral changes we observe will persist, as fear and media coverage subside once the lockdown is lifted and elements of daily life return to normal. These results suggest that the 
government should maintain a position to rapidly expand cash, medical, and food assistance, particularly in future lockdowns.

Further research is urgently required to understand what emergency relief programs could have the greatest impact for this population group; what factors would allow these behavioral changes, and in particular those relating to social distancing, to persist; the degree to which the risk of infection will impact the trust that people have in others as well as in authority figures, and the social, economic, and political consequences of those changes; and how the cumulative economic costs to this population group can be reversed over time, among others.

Despite the debilitating economic costs of the India lockdown, the potential value of the lives saved from the social distancing policies, and other behavioral changes that accompanied the lockdown, may also be large. This is especially the case in settings with underlying vulnerabilities, such as high levels of pre-existing health conditions, poor water and sanitation and other infrastructure, and high population densities. Ultimately, future decisions to implement extreme social distancing mandates will involve complex trade-offs between the costs of higher infection rates in a world that remains open, and the economic and non-economic costs in one that is periodically closed. A key factor influencing this trade-off is the extent to which people follow the public health directives. 


\section{References}

Afridi, Farzana, Amrita Dhillon, and Sanchari Roy. 2020. "How has Covid-19 crisis affected urban poor? Findings from a phone survey.” Ideas for India, April 23.

https://www.ideasforindia.in/topics/poverty-inequality/how-has-covid-19-crisis-affected-theurban-poor-findings-from-a-phone-survey.html.

Baker, Scott R., R.A. Farrokhnia, Steffen Meyer, Michaela Pagel, and Constantine Yannelis. 2020. "How Does Household Spending Respond to an Epidemic? Consumption During the 2020 COVID-19 Pandemic.” NBER Working Paper \#26949.

Bartik, Alexander, Marianne Bertrand, Zoë B. Cullen, Edward L. Glaeser, Michael Luca, and Christopher T. Stanton. 2020. "How Are Small Businesses Adjusting to COVID-19? Early Evidence from a Survey." NBER Working Paper \#26989.

Baylis, Patrick, Michael Greenstone, Kenneth Lee, and Harshil Sahai. 2020. "Pollution Masks and the Demand for Clean Air: Experimental Evidence from Delhi." Unpublished manuscript.

Bertrand, Marianne, Kaushik Krishnan, and Heather Schofield. 2020. "How are Indian households coping under the COVID-19 lockdown? 8 key findings." https://www.chicagobooth.edu/research/rustandy/blog/2020/how-are-indian-householdscoping-under-the-covid19-lockdown.

Burlig, Fiona, Steve Cicala, and Anant Sudarshan. 2020. "Impact of COVID-19 on Electricity Consumption and Particulate Pollution.” https://epic.uchicago.edu/area-of-focus/covid-19/.

Cherrie J.W., Andrew Apsley, Hilary Cowie, Susanne Steinle, William Mueller, Chun Lin, Claire J. Horwell, Anne Sleeuwenhoek, and Miranda Loh. 2018. "Effectiveness of Face Masks Used to Protect Beijing Residents Against Particulate Air Pollution." Occupational and Environmental Medicine 75(6): 446-452.

Clasen, Thomas, Sophie Boisson, Parimita Routray, Belen Torondel, Melissa Bell, Oliver Cumming, Jeroen Ensink, Matthew Freeman, Marion Jenkins, Mitsunori Odagiri, Subhajyoti Ray, Antara Sinha, Mrutyunjay Suar, Wolf-Peter Schmidt. 2014. "Effectiveness of a Rural Sanitation Programme on Diarrhoea, Soil-transmitted helminth Infection, and Child Malnutrition in Odisha, India: a Cluster-Randomised Trial." The Lancet Global Health 2(11): e645-e653.

Coibion, Olivier, Yuriy Gorodnichenko, and Michael Weber. 2020. "Labor Markets During the COVID-19 Crisis: A Preliminary View.” NBER Working Paper \#27017. 
Dupas, Pascaline, and Edward Miguel. 2017. "Impacts and Determinants of Health Levels in Low-Income Countries," Handbook of Field Experiments, (eds.) Esther Duflo and Abhijit Banerjee.

Gettleman, Jeffrey, and Suhasini Raj. 2020. "Powered by Fear, Indians Embrace Coronavirus Lockdown." New York Times, April 19.

https://www.nytimes.com/2020/04/19/world/asia/india-coronavirus-lockdown.html.

HT Correspondents. "Delhi observes Janta Curfew, city of nearly 20 million comes to a standstill." Hindustan Times, March 22. https://www.hindustantimes.com/india-news/delhiobserves-janta-curfew-city-of-nearly-20-million-comes-to-a-standstill/storycgaCHFWr91g0KzUeuw6INP.html.

Hussam, Reshmaan, Atonu Rabbani, Giovanni Reggiani, and Natalia Rigol. 2019. "Rational Habit Formation: Experimental Evidence from Handwashing in India." Harvard Business School Working Paper \#18-030.

Kremer, Michael, and Alix Peterson Zwane. 2007. "What Works in Fighting Diarrheal Diseases in Developing Countries? A Critical Review." The World Bank Research Observer 22(1): 1-24.

Langrish, Jeremy P., Xi Li, Shengfeng Wang, Matthew M. Y. Lee, Gareth D. Barnes, Mark R. Miller, Flemming R. Cassee, Nicholas A. Boon, Ken Donaldson, Jing Li, Liming Li, Nicholas L. Mills, David E. Newby, and Lixin Jiang. 2012. "Reducing Personal Exposure to Participate Air Pollution Improves Cardiovascular Health in Patients with Coronary Heart Disease." Environmental Health Perspectives 120(3): 367-372.

Maas, Paige, Andreas Gros, Laura McGorman, P. Alex Dow, Shankar Iyer, Wonhee Park, Chaya Nayak. "Facebook Disaster Maps: Aggregate Insights for Crisis Response \& Recovery" Proceedings of the 16th ISCRAM Conference. May 2019. https://research.fb.com/wpcontent/uploads/2019/04/iscram19_camera_ready.pdf

Marx, Benjamin, Thomas Stoker, and Tavneet Suri. 2013. "The Economics of Slums in the Developing World." Journal of Economic Perspectives 27(4): 187-210.

Pal, Alasdair, and Danish Siddiqui. 2020. "Special Report: India's migrant workers fall through cracks in coronavirus lockdown." Reuters, April 21. https://www.reuters.com/article/us-healthcoronavirus-india-migrants-spe/special-report-indias-migrant-workers-fall-through-cracks-incoronavirus-lockdown-idUSKBN2230M3.

Singh, Karan Deep, Vindu Goel, Hari Kumar, and Jeffrey Gettleman. 2020. "India, Day 1:

World's Largest Coronavirus Lockdown Begins." New York Times, March 25. https://www.nytimes.com/2020/03/25/world/asia/india-lockdown-coronavirus.html. 
Wu, Xiao, Rachel C. Nethery, M. Benjamin Sabath, Danielle Braun, and Francesca Dominici. 2020. "Exposure to air pollution and COVID-19 mortality in the United States: A nationwide cross-sectional study." medRxiv 2020.04.05.20054502.

Yadav, Anumeha. 2020. "India: Hunger and uncertainty under Delhi's coronavirus lockdown." Al Jazeera, April 19. https://www.aljazeera.com/news/2020/04/india-hunger-uncertainty-delhicoronavirus-lockdown-200418095253032.html.

Zheng, Ying-Ying, Yi-Tong Ma, Jin-Ying Zhang, and Xiang Xie. 2020. "COVID-19 and the cardiovascular system." Nature Reviews Cardiology 17: 259-260. 
Figure 1 - Impact of the lockdown on general intra-city movement in Delhi

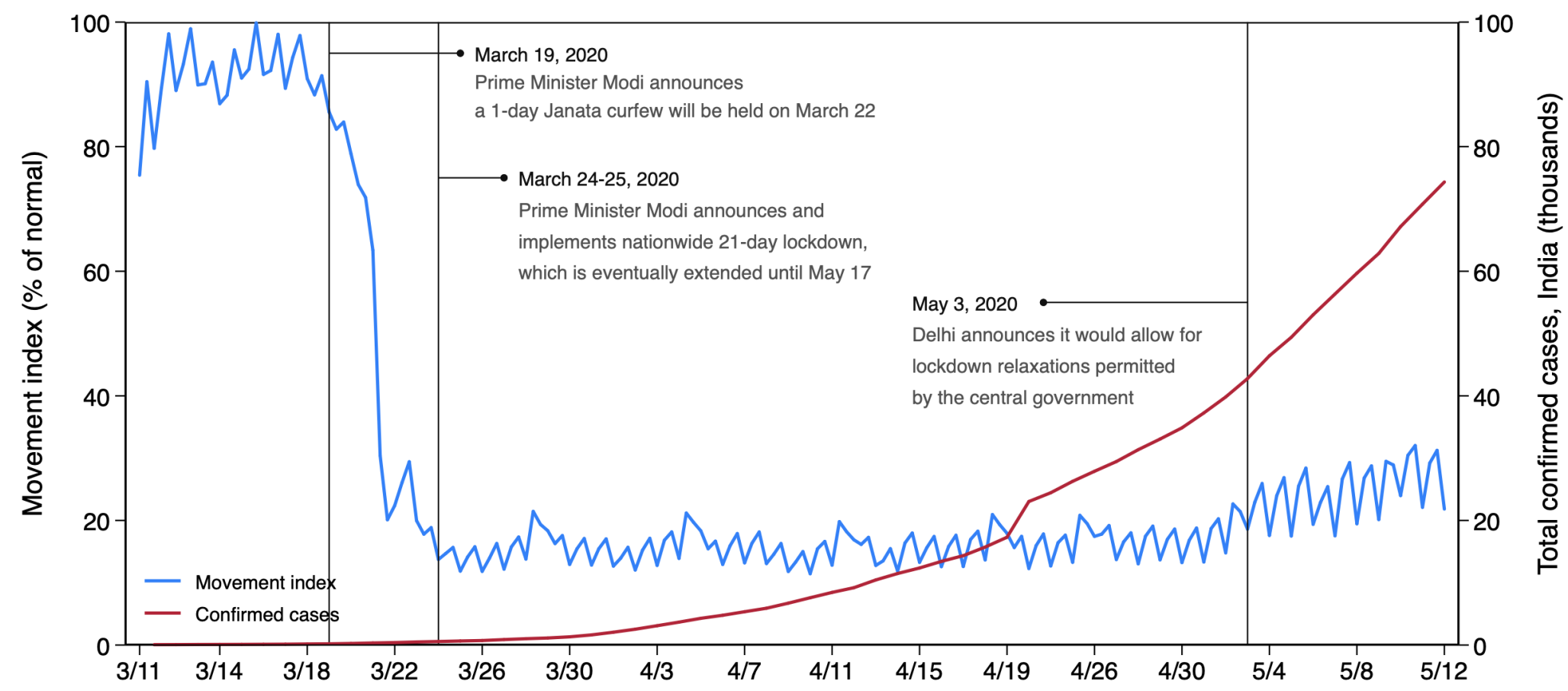

Source: Movement data is from Facebook Data for Good (Maas et al. 2019); Data on COVID-19 cases is from covid19india.org. Notes: We plot the movement index, which summarizes the intra-city movements of over two million users of the Facebook mobile app (which tracks locational data), against the total number of confirmed COVID-19 cases in India. Specifically, the movement index is constructed by calculating the average number of people who moved from one part of the city to another as a fraction of a baseline value for the period. Baseline values are defined as the average movement for the same time-of-day and day-of-week, in the 45 days preceding February 24, 2020, the start of available Facebook data. 
Figure 2 - Sample representativeness

A. Survey locations

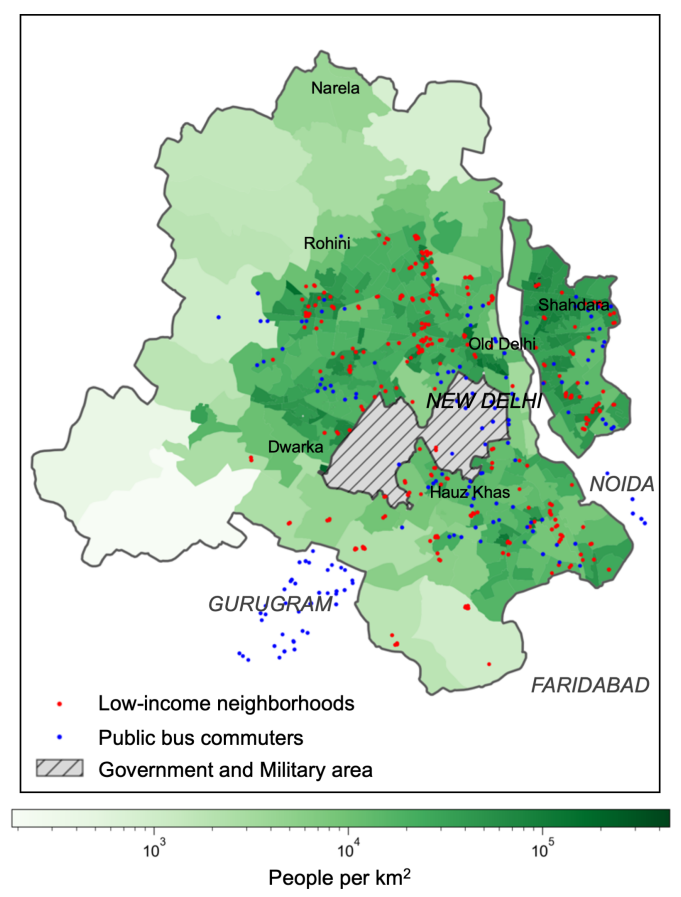

B. Pre-COVID-19 vs. NSS

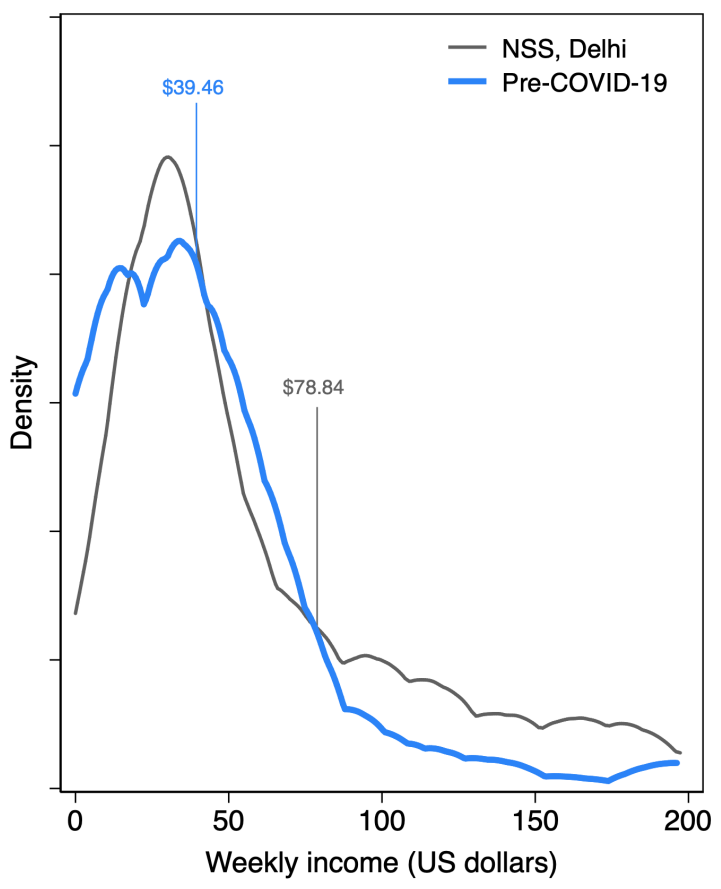

Source: Panel survey data collected between 2018 and 2020 from active participants in the labor force; 2011-12 National Sample Survey (NSS) 68th Round (Delhi sample).

Notes: In panel A, we display the approximate locations in which we enrolled respondents into our study. Red points show the locations of respondents in the low-income neighborhoods. Blue points show the locations of the public bus stops where we enrolled public bus commuters. In panel B, we plot and compare the baseline (i.e., pre-COVID-19) epanechnikov kernel (bandwidth 10) of income over the past week (converted to U.S. dollars) to that of the National Sample Survey (NSS) Delhi sample, after inflating the NSS data using CPI figures from the World Bank. Sample means are shown in the figure. 
Figure 3 - Impact of the lockdown on weekly income

A. Weekly income

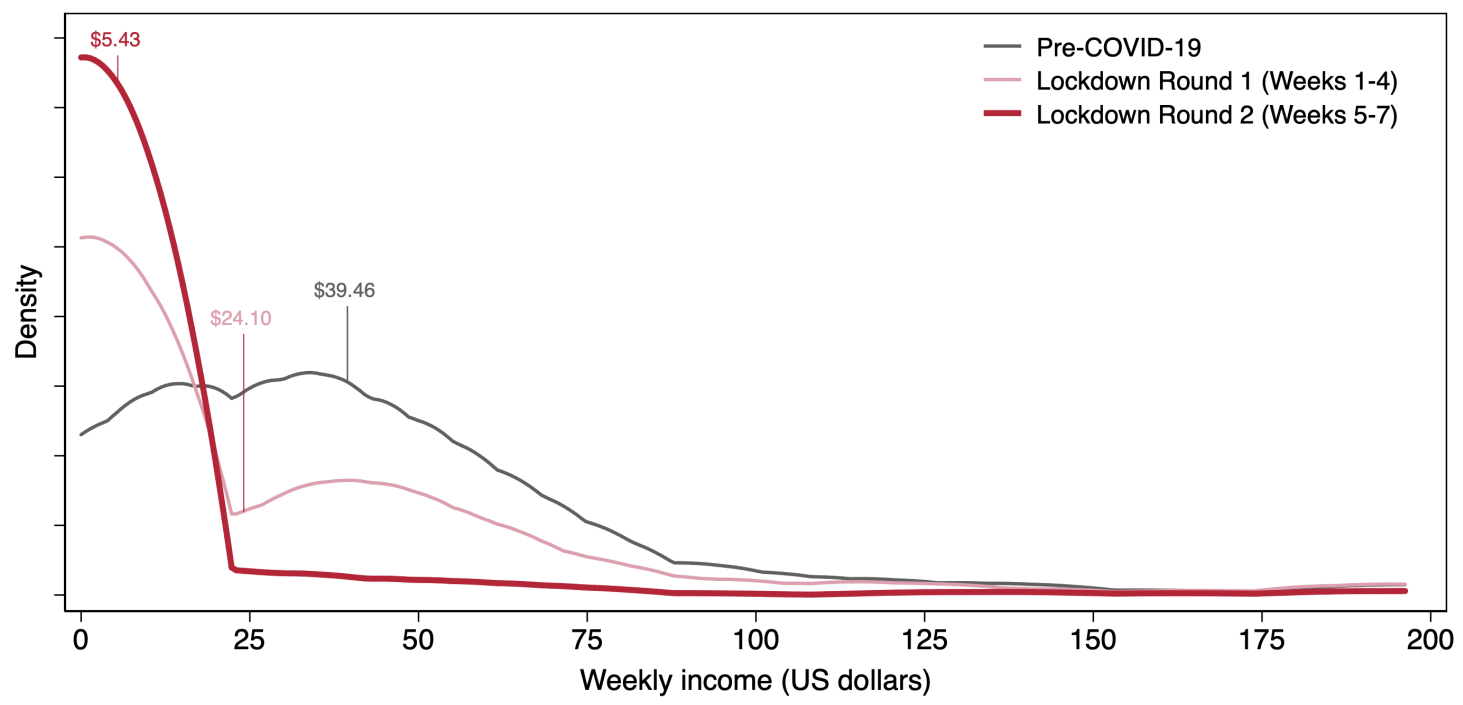

B. Change in weekly income, by occupation

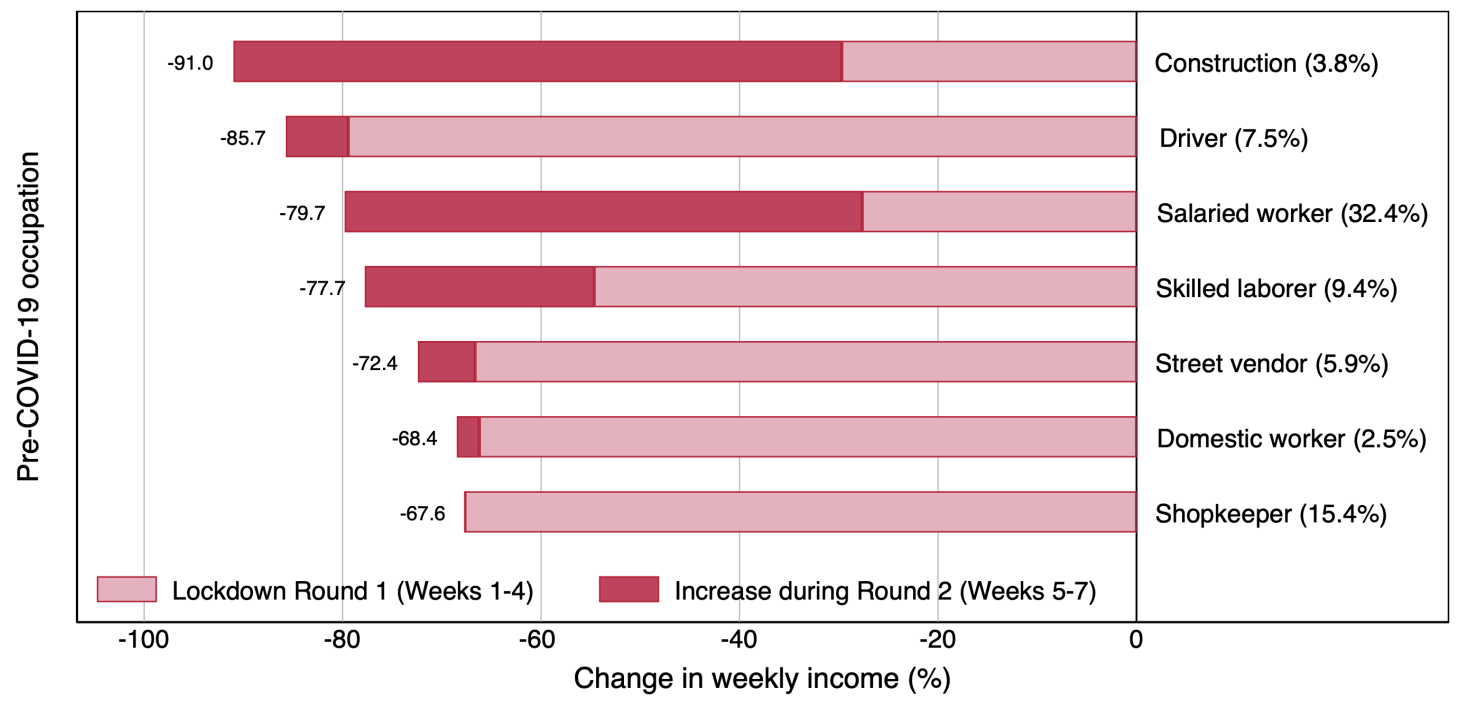

Source: Panel survey data collected between 2018 and 2020 from active participants in the labor force.

Notes: We plot epanechnikov kernels (bandwidth 10) of income over the past week (converted to U.S. dollars) at baseline ("Pre-COVID-19"), during weeks 1 to 4 of the COVID-19 lockdown ("Round 1"), and during weeks 5 to 7 ("Round 2"). Sample means are shown in the figure. In Panel B, we show the change in weekly income based on occupation categories recorded at baseline. For each occupation, we show the sample share in parantheses. 
Figure 4 - Impact of the lockdown on compliance with public health directives

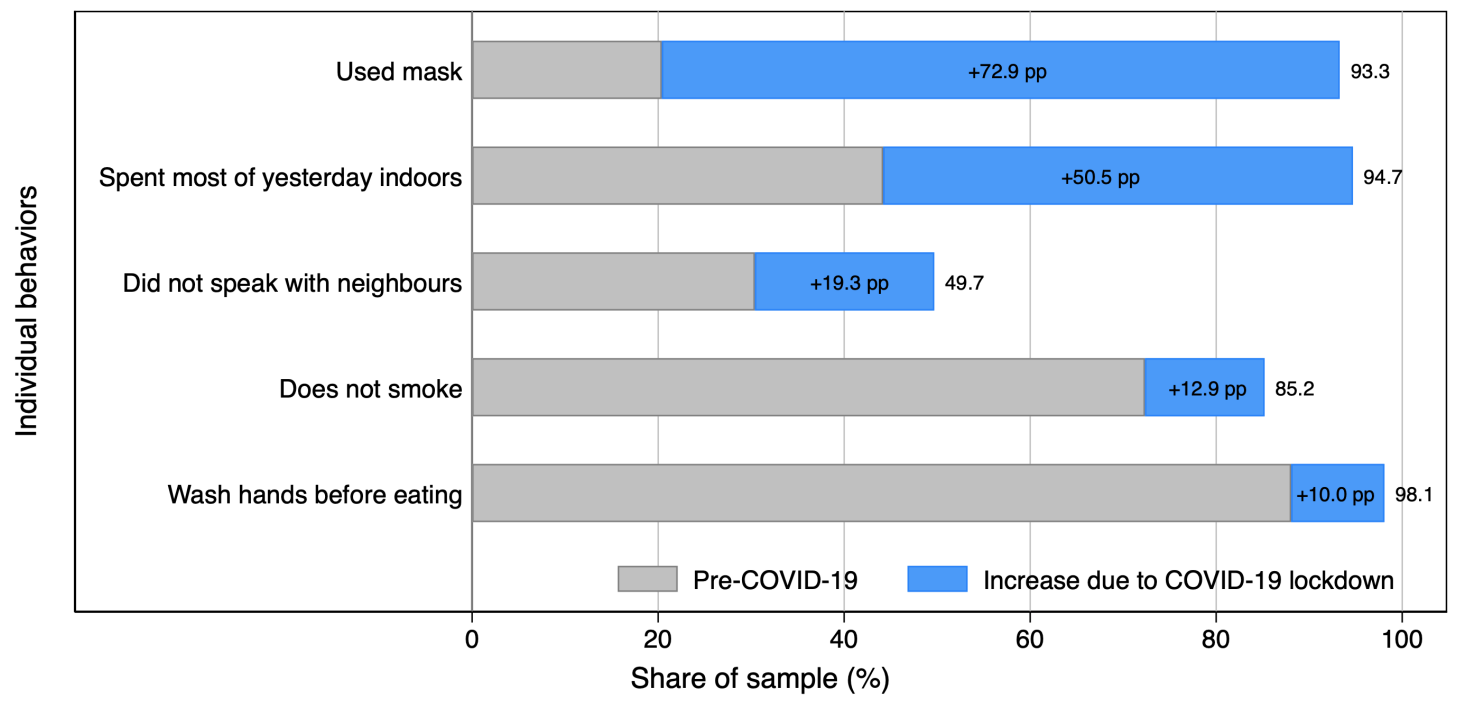

Source: Panel survey data collected between 2018 and 2020 from active participants in the labor force.

Notes: We present the effects of the lockdown on compliance with public health directives, averaging Round 1 and Round 2 data for individuals, if necessary. The outcome, "Did not speak with neighbors" is based on restrospective information collected in Round 2. For all other outcomes, we utilize data collected both at baseline and during the lockdown. 
Figure 5 - Media coverage as a potential factor driving the increase in mask usage, relative to during the peak air pollution period

A. Mask usage

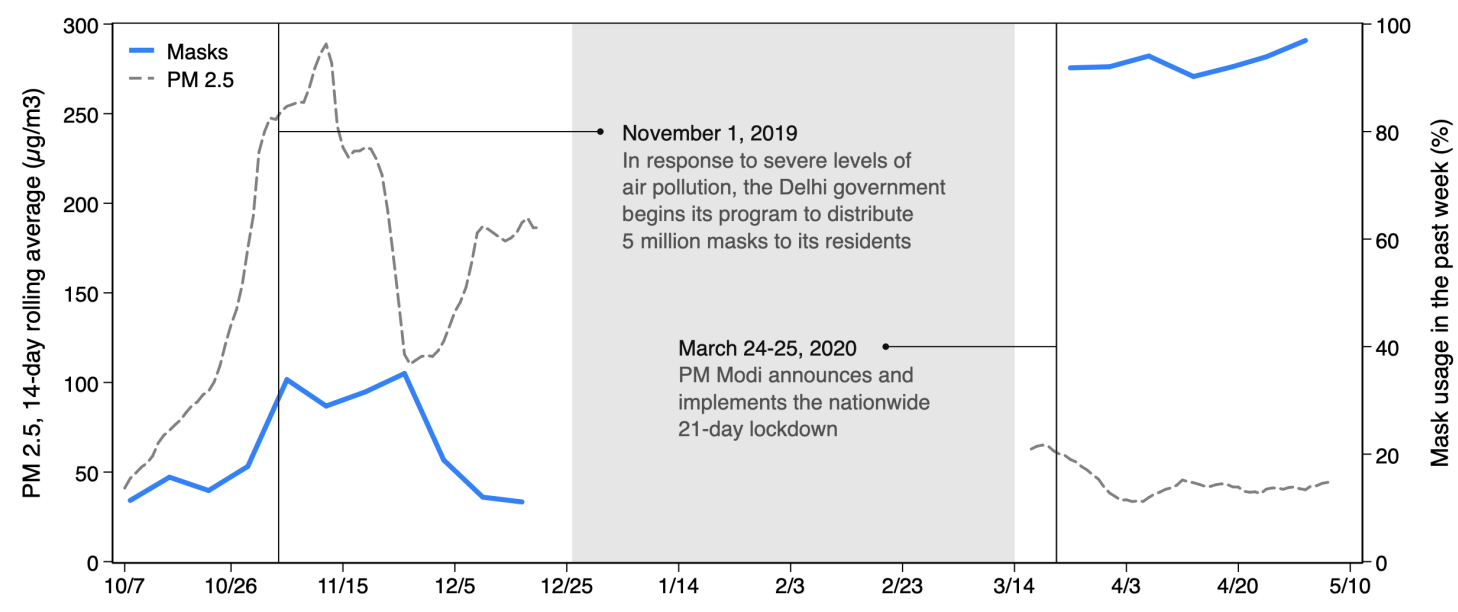

B. Media coverage

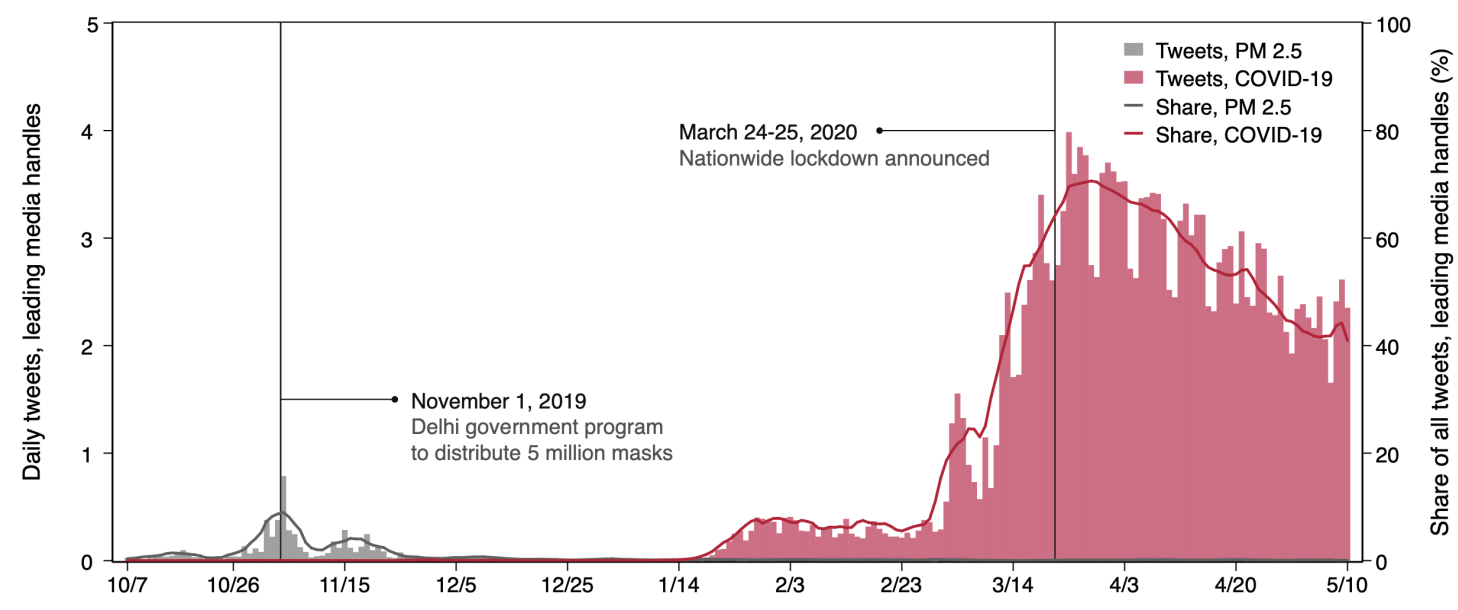

Source: Panel survey data collected between 2018 and 2020 from active participants in the labor force; U.S. Embassy, New Delhi, air pollution monitor; Twitter.

Notes: In Panel A, we plot the share of our sample reporting mask usage over the past week. The shaded region indicates the period in which there is no data. In Panel B, we plot Twitter data scraped from the top 50 media handles in Delhi. We focus on tweets containing the following keywords/phrases: pollution, delhi air, delhis air, delhi s air, airquality, air quality, airpurifier, air purifier, airemergency, air emergency, toxicair, toxic air, smog, stubbleburning, stubble burning, cropburning, crop burning, oddeven, odd even, pm2.5, pm25, pm10; and covid, corona, virus, pandemic, quarantine. At their peak, on March 27, 2020, COVID-19 tweets accounted for 70.6 percent of all tweets. In comparison, on November 3, 2020, air pollution tweets accounted for 8.8 percent of all tweets. 
Figure 6 - Negative income shocks and access to government benefits

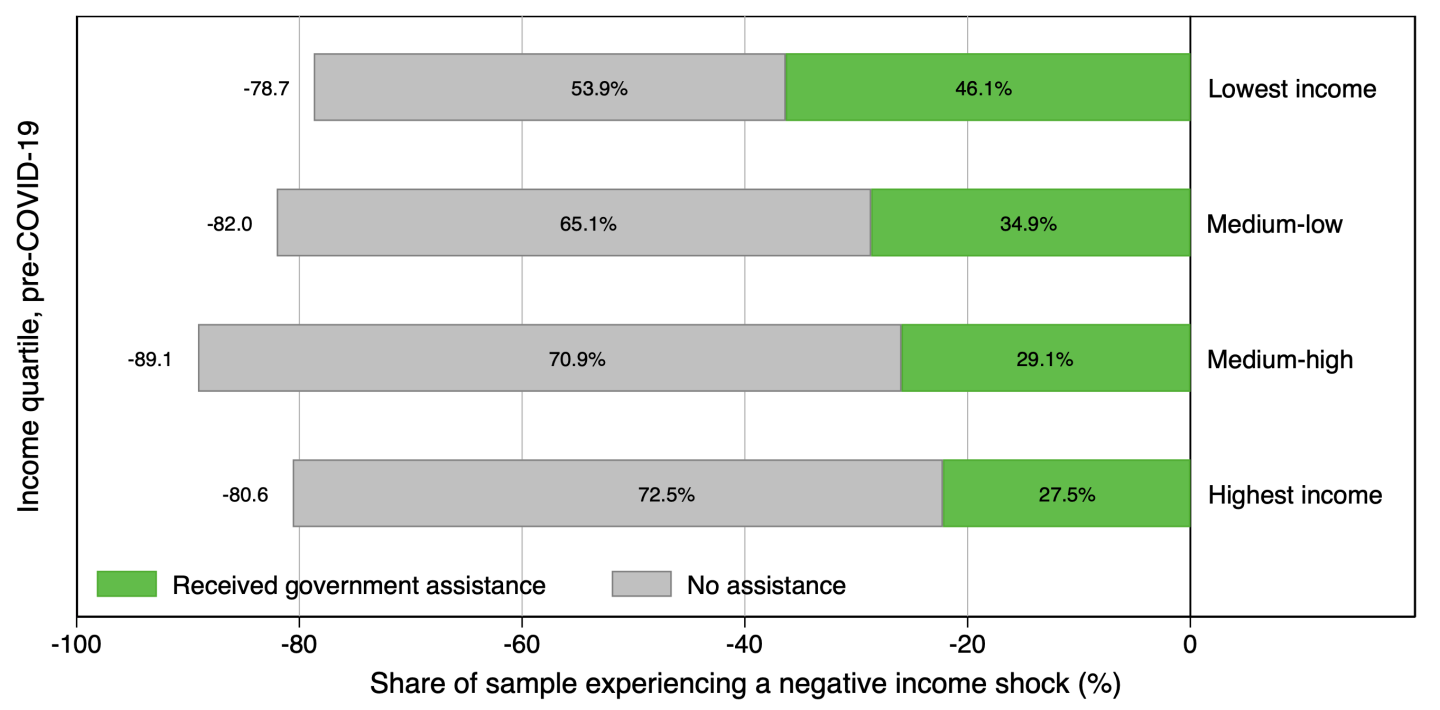

Source: Panel survey data collected between 2018 and 2020 from active participants in the labor force.

Notes: We present the share of each income quartile experiencing a negative shock to income, in addition to the share of those people who reported accessing government benefits during the lockdown. 95 percent of government benefits consisted of food assistance. 
Figure 7 - Non-economic costs experienced during the lockdown

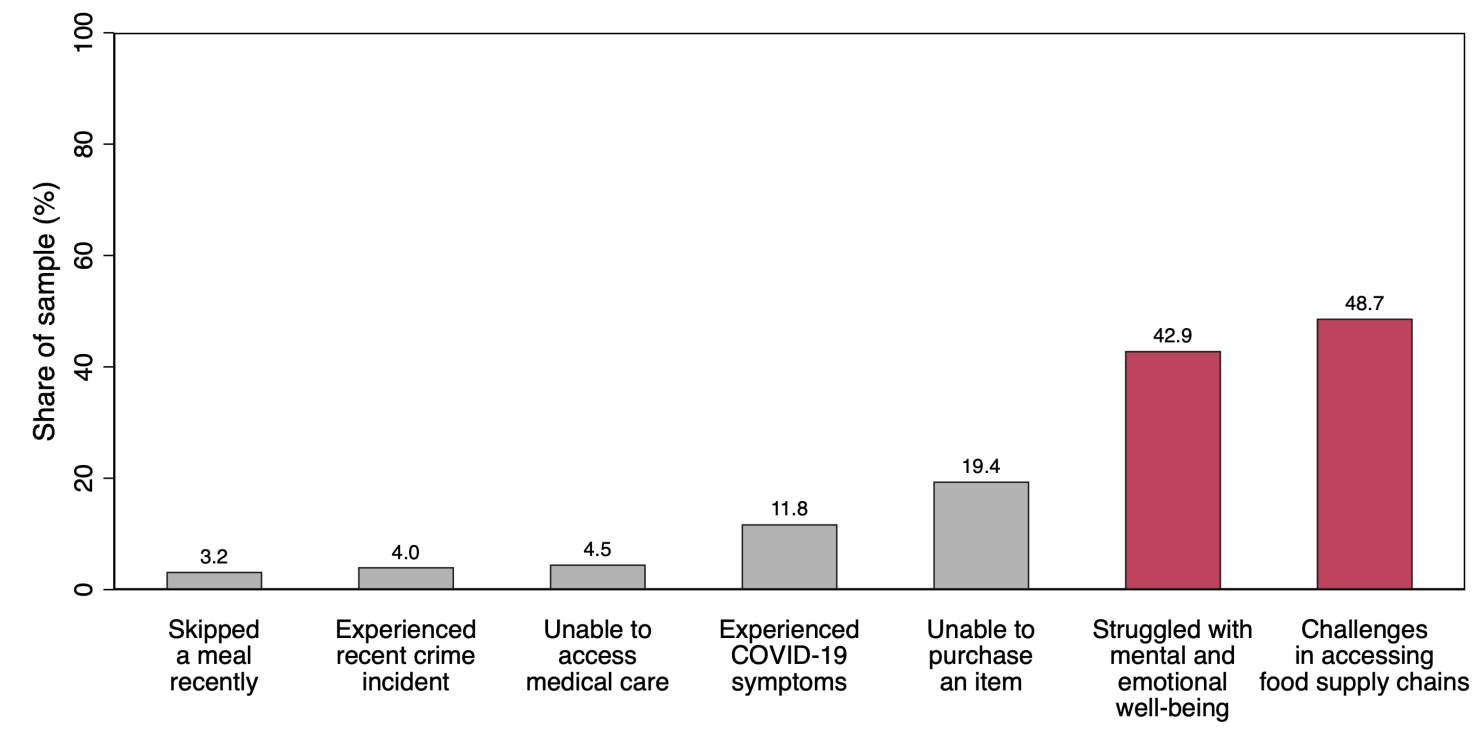

Source: Panel survey data collected between 2018 and 2020 from active participants in the labor force.

Notes: Data collection began on March 27, 2020, two days after the start of the lockdown. For each indicator, we plot the average result over seven weeks of data. The mental and emotional well-being outcome is defined as the share of individuals who reported feeling either depressed, bothered, that they lacked focus, or that everything was an effort, for the majority of the week leading up to the survey. Challenges accessing food supply chains include: mobility restrictions, inadequate quantities available, closed markets, and price shocks. 
Table 1 - Characteristics of active participants in the labor force

\begin{tabular}{|c|c|c|c|c|}
\hline & $\begin{array}{c}\text { Full sample } \\
\text { (1) }\end{array}$ & $\begin{array}{l}\text { Low-income } \\
\text { neighbor- } \\
\text { hoods } \\
(2)\end{array}$ & $\begin{array}{c}\text { Public bus } \\
\text { commuters } \\
\text { (3) }\end{array}$ & $\begin{array}{c}p \text {-value of } \\
\text { diff. } \\
(4)\end{array}$ \\
\hline \multicolumn{5}{|l|}{ Panel A: Demographic characteristics } \\
\hline Female $(\%)$ & 12.7 & 18.6 & 4.9 & $<0.01$ \\
\hline Age (years) & 35.4 & 35.8 & 34.8 & 0.11 \\
\hline Completed secondary school (\%) & 47.9 & 35.0 & 65.2 & $<0.01$ \\
\hline Household size & 5.1 & 5.4 & 4.8 & $<0.01$ \\
\hline Long-term Delhi resident (\%) & 87.3 & 94.7 & 77.2 & $<0.01$ \\
\hline \multicolumn{5}{|c|}{ Panel B: Common (primary) occupations } \\
\hline Salaried job (\%) & 32.4 & 18.8 & 50.6 & $<0.01$ \\
\hline Business (\%) & 15.4 & 18.2 & 11.6 & $<0.01$ \\
\hline Skilled labour (\%) & 9.4 & 10.5 & 7.9 & 0.10 \\
\hline Driver $(\%)$ & 7.5 & 8.2 & 6.7 & 0.32 \\
\hline Street vendor $(\%)$ & 5.9 & 8.3 & 2.7 & $<0.01$ \\
\hline Homemaker (\%) & 5.4 & 8.4 & 1.3 & $<0.01$ \\
\hline Construction worker (\%) & 3.8 & 4.9 & 2.4 & 0.01 \\
\hline Student (\%) & 3.4 & 2.5 & 4.5 & 0.04 \\
\hline Domestic worker $(\%)$ & 2.5 & 4.1 & 0.3 & $<0.01$ \\
\hline \multicolumn{5}{|l|}{ Panel C: Economic indicators } \\
\hline BPL card holder $(\%)$ & 19.7 & 22.6 & 15.4 & $<0.01$ \\
\hline Weekly individual income (USD) & 39.46 & 28.57 & 53.17 & $<0.01$ \\
\hline Weekly household income (USD) & 58.44 & 45.13 & 73.38 & $<0.01$ \\
\hline Government water source & 21.55 & 23.6 & 18.8 & 0.03 \\
\hline Distance to gov't assistance $(\mathrm{km})$ & 646.4 & 623.0 & 690.5 & 0.19 \\
\hline Sample size & 1,392 & 797 & 595 & \\
\hline
\end{tabular}

Notes: Active participants in the labor force are defined as those individuals who were employed in at least one survey round during the course of the study. Columns 2 and 3 report sample means for the slum residents and public bus commuters samples, respectively. Column 4 reports $p$-value of the difference between the means. In panel $\mathrm{C}$, we include sample means for BPL ("Below Poverty Line") card holder and Government water source (which captures whether the individual's primary drinking water source is a Delhi Jal Board tanker) as additional potential indicators of low-income status. 